\title{
Early life stress, HPA axis, and depression
}

\author{
Sandra Marcia de Carvalho Tofoli, Cristiane Von Werne Baes, Camila Maria Severi Martins and \\ Mario Juruena \\ Universidade de São Paulo, Ribeirão Preto, SP, Brazil
}

\begin{abstract}
Considerable evidence from various studies suggests a preeminent role for early adverse experiences in the development of psychopathology, especially depression. The most recent studies reviewed herein suggest that early life stressors are associated with an increased risk for mood disorders in adulthood. This review examines the emerging literature on the relationship between stress, hypothalamic-pituitary-adrenal (HPA) axis function, and depression and the role of early life stress as an important risk factor for HPA axis dysregulation. The most consistent findings in the literature show increased activity of the HPA axis in depression associated with hypercortisolemia and reduced inhibitory feedback. Moreover, HPA axis changes appear to be statedependent, tending to improve upon resolution of the depressive syndrome. Interestingly, persistent HPA hyperactivity has been associated with higher rates of relapse. These studies suggest that an evaluation of the HPA axis during antidepressant treatment may help identify patients who are at a higher risk for relapse. These findings suggest that this dysregulation of the HPA axis is partially attributable to an imbalance between glucocorticoid and mineralocorticoid receptors. Evidence has consistently demonstrated that glucocorticoid receptor function is impaired in major depression, but few studies have assessed the activity of mineralocorticoid receptors in depression. Thus, more studies are needed to elucidate this issue. Keywords: early life stress, childhood trauma, depression, treatment-resistant depression, hypothalamic-pituitary-adrenal axis.
\end{abstract}

Received 21 March 2011; received in revised form 11 June 2011; accepted 18 June 2011. Available on line 10 October 2011

\section{Introduction}

Psychological stress may change the internal homeostatic state of an individual. During acute stress, adaptive physiological responses occur, including increased adrenocortical hormone secretion, primarily cortisol. Whenever an acute interruption of this balance occurs, illness may result. Particularly interesting are psychological stress (i.e., stress in the mind) and the interactions with the nervous, endocrine, and immune systems.

A key problem in diagnosis is the fact that elaborate classification systems that exist today are solely based on subjective descriptions of symptoms. Such detailed phenomenology includes the description of multiple clinical subtypes, but no biological feature can distinguish one subtype from another. This approach

Sandra Marcia de Carvalho Tofoli, Cristiane Von Werne Baes, and Camila Maria Severi Martins, Department of Neuroscience and Behaviour, Faculty of Medicine Ribeirao Preto, University of Sao Paulo. Mario Juruena, Stress and Affective Disorders Programme, Department of Neurosciences and Behaviour, Faculty of Medicine Ribeirao Preto, University of Sao Paulo. Correspondence regarding this article should be directed to: Mario Juruena, Saúde Mental-USP, Av. Tenente Catão Roxo, 2650, Monte Alegre - Campus Universitário, Ribeirão Preto, SP CEP 14051-140, Brazil. E-mail: juruena@fmrp.usp.br states that the causes, development, and outcomes of disorders are determined by the relationships between psychological, social, and cultural factors and biochemistry and physiology. Biochemistry and physiology are not disconnected or different from the rest of our experiences and life events (Juruena, Marques, Mello, \& Mello, 2007).

\section{Early life stress}

The concept of early life stress is quite broad and includes the different traumatic experiences that occur during childhood and adolescence, which may have repercussions in adulthood. Among these are parental loss, separation from parents, childhood illness, family violence, and deprivation of food, clothing, shelter, and love.

In agreement with Bernstein et al. (1994), childhood maltreatment may be subdivided into the following domains:

1. Physical abuse: physical aggression by someone older, with the risk of or result of injury.

2. Emotional abuse: verbal aggression that affects the welfare or morale of the child or any conduct that humiliates, embarrasses, or threatens the child. 
3. Sexual abuse: any type of sexual contact or conduct between a child and someone older.

4. Emotional neglect: failure of caretakers to provide for basic emotional and psychological needs such as love, motivation, and support.

5. Physical neglect: failure of caretakers to provide for basic physical needs such as feeding, a home, security, supervision, and health (Bernstein et al., 1994, 2003).

Childhood maltreatment is a major social problem. It is a complex global phenomenon that does not respect boundaries of class, race, religion, age, or educational level and can occur both publicly and privately, resulting in serious physical injury or even death. Moreover, its psychological consequences can acutely affect a child's mental health well into adulthood (Friedrich, 1998; Cruz, Silva, \& Alves, 2007).

Early life stress is associated with a diverse range of psychiatric consequences. In children and adolescents, it increases the risk of behavioral problems, including internalizing and externalizing behavior. Internalizing refers to behavioral symptoms reflected by anxiety, depression, somatic complaints, and inhibition. Externalizing refers to behavioral symptoms reflected by aggression, delinquency, and increased activity level. Sexual behavior problems most likely fall into this domain (Friedrich, 1998).

Considerable evidence from various studies suggests a preeminent role for early adverse experiences in the development of mood and anxiety disorders. Child abuse and neglect can be perceived as agents for neurodevelopmental disruption and, depending on when it occurs, can cause serious neurological "scars" in some structures, which could make some individuals vulnerable to certain types of psychopathology, especially depression, posttraumatic stress disorder (PTSD), and substance abuse (Cohen, Brown, \& Smaile, 2001; Heim et al., 2000; Juruena, 2007).

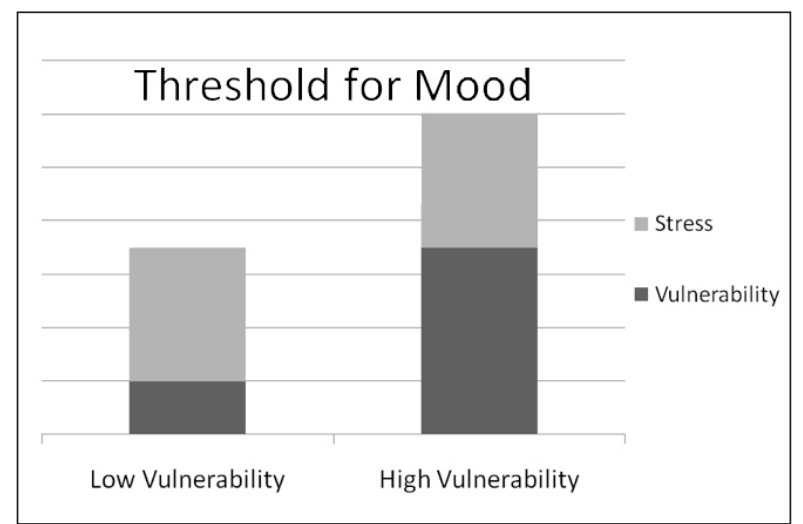

Figure 1. Model of vulnerability and stress. Adapted from Juruena, 2007.

\section{Psychoneuroendocrinology}

The maintenance of the internal homeostatic state of an individual is proposed to be based on the ability of circulating glucocorticoids to exert negative feedback on the secretion of hypothalamic-pituitary-adrenal (HPA) hormones through binding to mineralocorticoid receptors (MRs) and glucocorticoid receptors (GRs), limiting the vulnerability to diseases related to psychological stress in genetically predisposed individuals.

The HPA axis response to stress can be thought of as a mirror of the organism's response to stress: acute responses are generally adaptive, but excessive or prolonged responses can lead to deleterious effects. These deleterious effects, which may not be clinically apparent, have the potential to serve as endophenotypic markers of disease susceptibility (Juruena, Cleare, Bauer, \& Pariante, 2003; Mello et al., 2007). Because the HPA axis is activated in response to stressors, stressful events in early life may also have an etiologically significant role in the HPA abnormalities found in psychiatric disorders including Depression, Bipolar disorder, Anxiety disorders, Eating disorders, Schizophrenia, Substance abuse, Dissociative disorder, and PTSD, among other medical illnesses (Juruena, Cleare, \& Pariante, 2004; Mello et al., 2007).

According to Favarelli et al. (2010), the effect of childhood trauma on the development of psychopathology in adulthood is attributable to the HPA axis, which when activated during the developmental process would be permanently unstable, hyperstimulated, vulnerable, or dysfunctional (Favarelli et al., 2010). Similar findings were found in a recent study by Heim, Mletzko, Purselle, Musselman, and Nemeroff (2008) that assessed HPA axis function in men with and without major depression and childhood abuse. Abused men exhibited an increased cortisol response compared with non-abused men, regardless of the presence of major depression. Increased responses were associated with exposure to both sexual and physical abuse and were correlated with abuse severity. Their results suggested that childhood maltreatment is associated with impaired glucocorticoid-mediated feedback control of the HPA axis under stimulated conditions (Heim et al., 2008).

\section{Depression}

Approximately eight out of 10 people who experience a major depressive episode will have one or more further episodes during their lifetime (i.e., a recurrent major depressive disorder); therefore, early diagnosis and effective treatment are vital for reducing the effect of depression on the life of the individual, family, and community (Fava, Park, \& Sonino, 2006; Juruena et al., 2003). Studies estimate that the currently 
available antidepressant treatments are ineffective in 30$50 \%$ of treatment-resistant depression (TRD), i.e., are considered resistant those patients who do not respond to antidepressant treatment performed with a sufficient of time at an adequate dose.

Approximately $60 \%$ of cases of depressive episodes are preceded by exposure to stressors, especially psychosocial stressors (Joca, Padovan, \& Guimarães, 2003). Among the factors associated with depression in adulthood are exposure to childhood stressors such as the death of a parent or substitute, maternal deprivation, paternal abandonment, parental separation, and divorce (Zavaschi et al., 2002).

Maltreated children have a moderately increased risk of depression in adolescence and adulthood, which will partially mirror the family context in which the maltreatment occurred. Depression is common. Approximately one-quarter to one-third of maltreated children will meet the criteria for major depression by their late $20 \mathrm{~s}$, thus representing a substantial public health burden. For many of the affected individuals, the onset of depression begins in childhood, underscoring the importance of focusing on early intervention before the symptoms of depression appear in the abused and neglected children (Mello et al., 2009).

Hormones play a critical role in the development and expression of a wide range of behaviors. One aspect of the influence of hormones on behavior is their potential contribution to the pathophysiology of psychiatric disorders and the mechanism of action of psychotropic drugs, particularly in depression. Of the endocrine axes, the HPA axis has been the most widely studied. It plays a fundamental role in the response to external and internal stimuli, including psychological stressors. Abnormalities in the function of the HPA axis have been described in people who experience psychiatric disorders (Nemeroff, 1996).

Studies conducted in both animals and humans suggest that stress experienced during the early phases of development can induce persistent changes in the ability of the HPA axis to respond to stress in adulthood, increasing the susceptibility to depression (Glover \& O'Connor, 2002). Evidence suggests that neurochemical and molecular changes induced by stressful situations and depression trigger changes in the HPA axis. Findings derived from multiple lines of research have provided evidence that during depression, dysfunction of limbic structures, including the hypothalamus and hippocampus, results in hypersecretion of corticotropin-releasing factor (CRF), dehydroepiandrosterone, and vasopressin, which in turn determines HPA activation. A flaw in this system caused by factors such as excessive stress, high glucocorticoid levels, social isolation, and depressive symptoms results in difficulty adapting to stress and can predispose the individual to depression by impairing hippocampal serotonergic neurotransmission (Holsboer, 2000; Joca et al., 2003; Juruena et al., 2004; Figure 2).

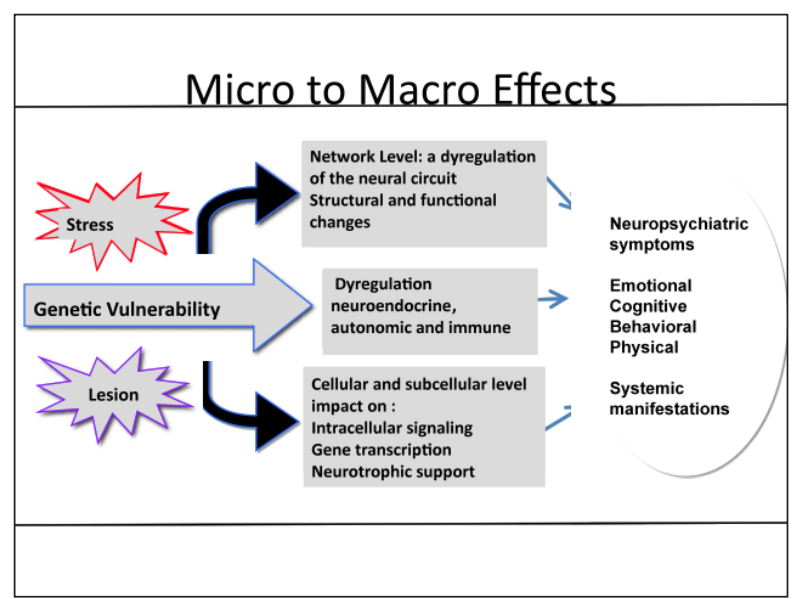

Figure 2. Micro and macro stress in individuals with genetic vulnerability and its relationship to neuropsychiatric symptoms. Adapted from Juruena et al., 2003.

Hyperactivity of the HPA axis in major depression is one of the most consistent findings in psychiatry. A significant percentage of patients with major depression have been shown to exhibit increased concentrations of cortisol (i.e., the endogenous glucocorticoid in humans) in plasma, urine, and cerebrospinal fluid, an exaggerated cortisol response to adrenocorticotropic hormone, and an enlargement of both the pituitary and adrenal glands (Nemeroff, 1996). In the past several years, many studies of the HPA axis in depressed patients have been published. A new perspective for these old findings of hypercortisolemia appears to have been generated. Hypercortisolemia is apparently linked to some specific cases of depression, depending on the type and severity of the illness, genotype, history of stress during childhood, and possibly resilience, leading us to hypothesize that an endophenotype may exist that is prone to depression (Mello et al., 2007). Previous studies have described impaired HPA negative feedback, leading to hypercortisolemia, such as in melancholic depression (Nemeroff, 1996; Gold, Goodwin, \& Chrousos, 1988). In addition to melancholic depression, a spectrum of other conditions may be associated with increased and prolonged activation of the HPA axis, including anorexia nervosa with and without malnutrition, obsessive-compulsive disorder, panic disorder, anxiety, chronic active alcoholism, alcohol and narcotic withdrawal, poorly controlled diabetes mellitus, and hyperthyroidism (Tsigos \& Chrousos, 2002). Another group of states is characterized by hypoactivation of the stress system, rather than sustained activation, in which chronically reduced secretion of CRF may result in pathological hypoarousal and enhanced HPA negative feedback. Patients with PTSD, atypical depression, seasonal depression, and chronic fatigue syndrome fall into this category (Kellner \& Yehuda, 1999; Juruena et al., 2004; Juruena \& Cleare, 2007; Table 1). 
Table 1 - States associated with hyperactivation or hypoactivation of the HPA axis

\begin{tabular}{|c|c|c|}
\hline Increased HPA axis activity & Decreased HPA axis activity & Disrupted HPA axis activity \\
\hline Severe chronic disease & Atypical depression & Cushing syndrome \\
\hline Melancholic depression & Seasonal depression & Glucocorticoid deficiency \\
\hline Anorexia nervosa & Chronic fatigue syndrome & Glucocorticoid resistance \\
\hline Obsessive-compulsive disorder & Fibromyalgia & \\
\hline Panic disorder & Hypothyroidism & \\
\hline Chronic excessive exercise & Adrenal suppression & \\
\hline Malnutrition & Post glucocorticoid therapy & \\
\hline Diabetes mellitus & Posttraumatic stress disorder & \\
\hline Hyperthyroidism & Nicotine withdrawal & \\
\hline Central obesity & Postpartum & \\
\hline Childhood maltreatment & Menopause & \\
\hline Pregnancy & Rheumatoid arthritis & \\
\hline
\end{tabular}

HPA, hypophysis-pituitary-adrenal. Adapted from Juruena et al., 2004.

One of the mechanisms thought to be involved in HPA axis hyperactivity in depression is impaired feedback inhibition of the HPA axis by circulating glucocorticoids (Pariante \& Miller 2001). This impaired feedback inhibition has been demonstrated in depressed patients in various studies, many conducted in the 1970s and 1980s (Juruena et al., 2009; Juruena, Pariante, Papadopoulos, \& Cleare, 2010; Ribeiro, Tandon, Grunhaus, \& Greden, 1993). These abnormalities appear to be related to changes in the ability of circulating glucocorticoids to exert negative feedback on the secretion of HPA hormones through binding to MRs and GRs in HPA tissue (de Kloet, Vreugdenhil, Oitzl, \& Joels, 1998; Gold et al., 1988; Juruena et al., 2003; Nemeroff, 1996; Figure 3). The first study of hypercortisolemia in depressed patients was published in the 1970s and found that severely depressed patients exhibited non-suppression in the dexamethasone suppression test (DST). In the DST, a high proportion of patients with various affective disorders had elevated cortisol levels, thus negating the suppressive effect of dexamethasone. Unfortunately, dexamethasone has pharmacodynamic and pharmacokinetic features that are very distinct from those of the human endogenous glucocorticoid cortisol. These features, together with the low sensitivity of the DST in detecting patients with major depression, have significantly limited the use of this test in routine clinical and research practice. Recently, a suppressive test that uses another synthetic glucocorticoid, prednisolone, was developed by Pariante et al. (2002). This test has a higher affinity for MRs and, therefore, should probe both receptors (Juruena et al., 2006; Juruena et al., 2009, 2010; Pariante et al., 2002).

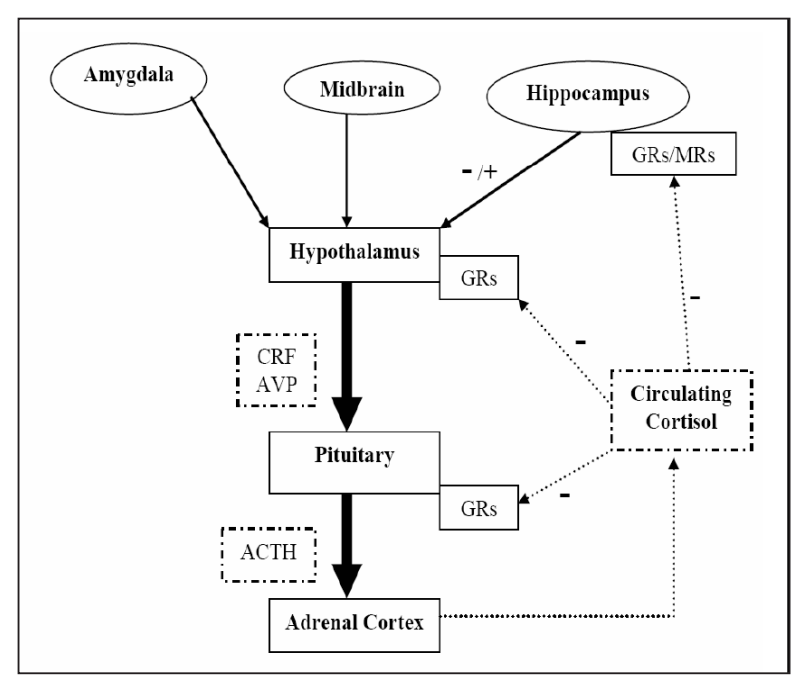

Figure 3. Schematic diagram of the hypothalamic-pituitaryadrenal (HPA) axis that describes the regulation and negative feedback (-) of cortisol via glucocorticoid receptors (GRs) and mineralocorticoid receptors (MRs). Adapted from Juruena et al., 2004. 


\section{Prediction of response}

Generally, HPA axis changes appear in chronic depressive and more severe episodes. Moreover, HPA axis changes appear to be state-dependent, tending to improve upon resolution of the depressive syndrome (Holsboer, 2000). Interestingly, persistent HPA hyperactivity has been associated with higher rates of relapse (Juruena et al., 2009; Ribeiro et al., 1993; Zobel, Yassouridis, Frieboes, \& Holsboer, 1999). Studies conducted in patients who received a range of antidepressants have shown that those who fail to show a normalization of post-dexamethasone cortisol levels tend to have a worse outcome with regard to re-hospitalization, suicide, and the recurrence of depression (Ribeiro et al., 1993). Recent reports have described a prospective study of the relationship between the results of the dexamethasone/CRF test and clinical outcome. Specifically, Zobel et al. (1999; Zobel et al., 2001) described a cohort of patients who were subjected to the dexamethasone/CRF test on two separate occasions: within 1 week after admission (or after the start of the first antidepressant treatment) and a few days before discharge. The patients were then followed up for 6 months after discharge. The study found that the patients who exhibited increased cortisol levels after the dexamethasone/CRF test between admission and discharge tended to relapse during the follow-up period, whereas those who showed a decrease in post-dexamethasone/CRF cortisol levels tended to remain clinically stable during the followup period (Zobel et al., 1999, 2001). Therefore, these studies suggest that the evaluation of the HPA axis during antidepressant treatment may help identify patients who have a higher risk of relapse (Juruena et al., 2009; Zobel et al., 1999).

\section{Synthesis}

Studies of the association between early life stress and psychiatric disorders should be evaluated carefully. No consensus has been reached in the literature regarding the concept of early life stress, and the respondents in these studies likely underestimated or overestimated the frequency/intensity of events. Much descriptive work has been published on the relationship between adult psychopathology and early adversities such as parental loss in childhood, inadequate parental care, divorce, "affectionless" or dysfunctional parenting, childhood physical and sexual abuse, and other childhood traumas. Importantly, mood disorders such as depression are most associated with the occurrence of early life stress subtypes. The results of existing studies suggest the importance of preventing early life stress and its consequences in both the short and long terms. Intervention at an early stage can reduce the likelihood of developing health problems in the long term and re-victimization in adulthood. Furthermore, early interventions may reduce the burden of public spending on healthcare for abused individuals.

The more recent studies reviewed in the present paper suggest that early life stressors are associated with an increased risk for mood disorders in adulthood. This review examined the emerging literature concerning the relationship between stress, HPA axis function, and depression and early life stress as an important risk factor for HPA axis dysregulation. The most consistent findings in the literature show increased activity of the HPA axis in depression associated with hypercortisolemia and reduced inhibitory feedback. These findings suggest that this dysregulation of the HPA axis is partially attributable to an imbalance between GRs and MRs. Evidence has consistently demonstrated that GR function is impaired in major depression, resulting in reduced GR-mediated negative feedback on the HPA axis, but few studies have assessed the activity of MRs in depression. Thus, although a few studies suggested that MR activity remains intact or is possibly oversensitive to compensate for reduced GR function in depressed patients, more studies are needed to elucidate this issue (Young, Lopez, MurphyWeinberg, Watson, \& Akil, 2003; Juruena et al., 2009, 2010; Pariante et al., 2001; Pariante, 2006).

\section{Conclusion}

Social and physical environments have an enormous impact on our physiology and behavior, and they influence the process of adaptation or allostasis. At the same time that our experiences change our brain and thoughts (i.e., changing our mind), we change our neurobiology. Although disturbances in the HPA axis are an important factor in the etiology of depression and severe treatment resistance, very little is known about the neurobiology of these disorders. Therefore, psychometric assessment that quantifies the level of early life stress, recent stress, the evolution of affective symptoms and diagnosis, and neuroendocrine activity is essential. Childhood stressful events and HPA axis overactivity in adulthood are not specific to depressive states, but several studies have linked these conditions. As demonstrated in this review, early life stress leads to permanent changes in the HPA axis and may lead to the development of depression in adulthood. Considering the importance of early detection of violence in childhood and adolescence, to prevent the development of severe and disabling psychiatric disorders in adulthood, further research is needed to elucidate the mechanisms involved in the association between early stress and the development of psychopathology in adulthood. 


\section{References}

Bernstein, D.P., Fink, L., Handelsman, L., Foote, J., Lovejoy, M., Wenzel, K., Sapareto, E., \& Ruggiero, J. (1994). Initial reliability and validity of a new retrospective measure of child abuse and neglect. American Journal of Psychiatry, 151, 1132-1136.

Bernstein, D.P., Stein, J.A., Newcomb, M.D., Walker, E., Pogge, D., Ahluvalia, T., ...Zule, W. (2003). Development and validation of a brief screening version of the Childhood Trauma Questionnaire. Child Abuse and Neglect, 27, 169-190.

Cohen, P., Brown, J., \& Smaile, E. (2001). Child abuse and neglect and the development of mental disorders in the general population. Development and Psychopathology, 981-999.

Cruz, D.M.C., Silva, J.T., \& Alves, H.C. (2007). Evidências sobre violência e deficiência: implicações para futuras pesquisas. Revista Brasileira de Educação Especial, 13(1), 131-146.

de Kloet, E.R., Vreugdenhil, E., Oitzl, M.S., \& Joels, M. (1998). Brain corticosteroid receptor balance in health and disease. Endocrine Reviews, 19, 269-301.

Faravelli, C., Amedei, S.G., Rotella, F., Faravelli, L., Palla, A., Consoli, G., ...dell'Osso, M.C. (2010). Childhood traumata, Dexamethasone Suppression Test and psychiatric symptoms: a trans-diagnostic approach. Psychological Medicine, 40, 2037-2048.

Fava, G.A., Park, S.K., \& Sonino, N. (2006). Treatment of recurrent depression. Expert Review of Neurotherapeutics, 6, 1735-1740.

Friedrich, W. (1998). Behavioral manifestations of child sexual abuse. Child Abuse and Neglect, 22, 523-531.

Glover, V., \& O'Connor, T.G. (2002). Effects of antenatal stress and anxiety: implications for development and psychiatry. British Journal of Psychiatry, 180, 389-391.

Gold, P.W., Goodwin, F.K., \& Chrousos, G.P. (1988). Clinical and biochemical manifestation of depression: relation to the neurobiology of stress. New England Journal of Medicine, 319, 413-420.

Heim, C., Mletzko, T., Purselle, D., Musselman, D.L., \& Nemeroff, C.B. (2008). The dexamethasone/corticotropin-releasing factor test in men with major depression: role of childhood trauma. Biological Psychiatry, 639, 398-405.

Heim, C., Newport, D.J., Heit, S., Graham, Y.P., Wilcox, M., Bonsall, R., Miller, A.H., \& Nemeroff, C.B. (2000). Pituitary-adrenal and autonomic responses to stress in women after sexual and physical abuse in childhood. Journal of the American Medical Association, 284, 592-597.

Holsboer, F. (2000). The corticosteroid receptor hypothesis of depression. Neuropsychopharmacology, 23, 477-501.

Joca, S.R.L., Padovan, C.M., \& Guimarães, F.S. (2003). Estresse, depressão e hipocampo. Revista Brasileira de Psiquiatria, 25(Suppl. 2), 46-51.

Juruena, M.F. (2007). The neurobiology of treatment resistant depression: role of the hypothalmic-pituitary-adrenal axis and glucocorticoid and mineralocorticoid receptor function. $\mathrm{PhD}$ thesis, University of London, King's College London, Institute of Psychiatry.

Juruena, M.F., \& Cleare, A.J. (2007). Overlap between atypical depression, seasonal affective disorder and chronic fatigue syndrome. Revista Brasileira de Psiquiatria, 29(Suppl. 1), S19-S26.

Juruena, M.F, Cleare, A.J., Bauer, M.E., \& Pariante, C.M. (2003) Molecular mechanism of GR sensitivity and relevance for affective disorders. Acta Neuropsychiatrica, 15, 354-367.

Juruena, M.F., Cleare, A.J., Papadopoulos, A.S., Poon, L., Lightman, S., \& Pariante, C.M. (2006). Different responses to dexamethasone and prednisolone in the same depressed patients. Psychopharmacology, 189, 225-235.

Juruena, M.F., Cleare, A.J., \& Pariante, C.M. (2004). The hypothalamic pituitary adrenal axis, glucocorticoid receptor function and relevance to depression. Revista Brasileira de Psiquiatria, 26, 189-201.

Juruena, M.F., Marques, A.H., Mello, A.F., \& Mello, M.F. (2007). A paradigm for understanding and treating psychiatric illness. Revista Brasileira de Psiquiatria, 29(Suppl. 1), S1-S2.

Juruena, M.F., Pariante, C.M., Papadopoulos, A., \& Cleare, A.J. (2010). The development and application of the prednisolone suppression test in psychiatry: a novel tool for assessing glucocorticoid and mineralocorticoid receptor function. Mind \& Brain, the Journal of Psychiatry, 1(1), 115-122.

Juruena, M.F., Pariante, C.M., Papadopoulos, A.S., Poon, L., Lightman, S., \& Cleare, A.J. (2009). Prednisolone suppression test in depression: prospective study of the role of HPA axis dysfunction in treatment resistance. British Journal of Psychiatry, 194, 342-349.

Kellner, M., \& Yehuda, R. (1999). Do panic disorder and posttraumatic stress disorder share a common psychoneuroendocrinology? Psychoneuroendocrinology, 24, 485-504.

Mello, M.F., Faria, A.A., Mello, A.F., Carpenter, L.L., Tyrka, A.R., \& Price, L.H. (2009). Maus-tratos na infância e psicopatologia no adulto: caminhos para a disfunção do eixo hipotálamo-pituitáriaadrenal. Revista Brasileira de Psiquiatria, 31(Suppl. 2), 41-48.

Mello, A.F., Juruena, M.F., Pariante, C.M., Tyrka, A.R, Price, L.H., Carpenter, L.L., \& Del Porto, J.A. (2007). Depression and stress is there an endophenotype? Revista Brasileira de Psiquiatria, 29(Suppl. 1), S13-S18.

Nemeroff, C.B. (1996). The corticotropin-releasing factor (CRF) hypothesis of depression: new findings and new directions. Molecular Psychiatry, 1(4), 336-342.

Pariante, C.M. (2006). The glucocorticoid receptor: part of the solution or part of the problem? Journal of Psychopharmacology, 20(4 Suppl.), 79-84.

Pariante, C.M., \& Miller, A.H. (2001). Glucocorticoid receptors in major depression: relevance to pathophysiology and treatment Biological Psychiatry, 49, 391-404.

Pariante, C.M., Papadopoulos, A.S., Poon, L., Checkley, S.A., English, J., \& Kerwin, R.W., Lightman, S. (2002). The novel prednisolone suppression test for the hypothalamic-pituitary-adrenal axis. Biological Psychiatry, 51, 922-930.

Ribeiro, S.C.M., Tandon, R., Grunhaus, L., \& Greden, J.F. (1993) The DST as a predictor of outcome in depression: a meta-analysis. American Journal of Psychiatry, 150, 1618-1629.

Souery, D., Oswald, P., Massat, I., Bailer, U., Bollen, J., Demyttenaere K., Mendlewicz, J. (2007). Clinical factors associated with treatment resistance in Major Depressive Disorder: Results from a European Multicenter Study. Journal Clinic Psychiatry, 68, 1062-1070.

Tsigos, C., \& Chrousos, G.P. (2002). Hypothalamic-pituitary-adrenal axis, neuroendocrine factors and stress. Journal of Psychosomatic Research, 53, 865-871.

Young, E.A., Lopez, J.F., Murphy-Weinberg, V., Watson, S.J., \& Akil, H. (2003). Mineralocorticoid receptor function in major depression. Archives of General Psychiatry, 60(1), 24-28.

Zavaschi, M.L.S., Satler, F., Poester, D., Vargas, C.F., Piazenski, R., Rohde, L.A.P., \& Eizirik, C.L. (2002). Associação entre trauma por perda na infância e depressão na vida adulta. Revista Brasileira de Psiquiatria, 24, 189-195.

Zobel, A.W., Nickel, T., Sonntag, A., Uhr, M., Holsboer, F., \& Ising, M. (2001). Cortisol response in the combined dexamethasone/CRH test as predictor of relapse in patients with remitted depression. a prospective study. Journal of Psychiatric Research, 35, 83-94.

Zobel, A.W., Yassouridis, A., Frieboes, R.M., \& Holsboer, F. (1999). Prediction of medium-term outcome by cortisol response to the combined dexamethasone-CRH test in patients with remitted depression. American Journal of Psychiatry, 156, 949-951. 